ANALYSIS OF MODELS WITH HOMOZYGOTE $\times$ HETEROZYGOTE MATINGS

\author{
SAMCEL KARLIN AND MARCUS W. FELDMAN* \\ Department of Mathematics Stanford University, Stanford, California 94305 \\ Received October 3, 1967
}

THE general genetic incompatibility model can be described by specifying which, among the types of mating considered possible, are allowed to occur. This encompasses the original self sterility mechanisms in Nicotiana (EAST and MANGelsdorf 1925), the "pollen elimination" and "zygote elimination" models of Finney (1952), heterostyly in Primula (Bodmer 1960, Moran 1962) and those models involving assortative matings as proposed by Watterson (1959), NAYLOR (1962) and Workman (1964).

Since these incompatibility systems have been found so widely in nature, the question of what polymorphic equilibria can be expected from a given model is quite an important one. However, the stability of, and rate of approach to these equilibria are just as important from an evolutionary point of view. Historically, it has been the practice to demonstrate the stability or otherwise of genetic systems such as incompatibility models by local linear analysis (see OwEN 1953 and Workman 1964). Mathematically, the fact that such a local linear analysis produces one eigenvalue greater than unity is not sufficient to guarantee instability. In fact it may merely mean that a trajectory can move away from the fixed point in one direction and return along another. This fact seems to have been misunderstood in the literature.

Recently high speed computation has been used in a large number of models to show that starting from special initial genotype frequencies, some fixed point can be reached. In this note we consider certain incompatibility systems introduced by Finney and more extensively pursued by Scudo (1964) as a basis for some models of polygenic sex determination. These models fit quite comfortably into the broader class of incompatibility systems mentioned above. Scudo has determined the equilibrium points (or in some cases families of equilibrium points) and, using numerical methods, has verified that for special initial genotype frequencies some of these equilibrium points could be reached. In this paper we have chosen some of Scuno's models and attempted to ascertain their complete equilibrium behavior; that is the determination of the equilibria themselves, the rates of approach and domains of attraction to the equilibria. Our objective is to carry out a complete mathematical analysis of these models highlighting certain qualitative implications which may have some evolutionary significance and which are not obvious from numerical computations. These conclusions are

\footnotetext{
- Research supported in part under contract N0014-67-A-0112-0015 and grant NIH 10452 at Stanford University, Stanford, California.

Genetics 59: 105-116 May 1968.
} 
further reinforced by those obtained in the companion paper (KARLIN and FELDMaN 1968) in which we consider some related models due to Workman and NAYLOR, as well as their "zygote elimination" modifications.

\section{MODELS AND RESULTS}

The incompatibility mechanism used here can be viewed in two ways. First, one can suppose that all conceivable matings take place, but that the offspring produced from certain specified matings are inviable. Second, the genotypes can be considered to be partitioned into two sets, with matings possible only between individuals in different sets although at random within this restriction. In the terminology of FINNEY (1952) the models are of the zygote elimination type. For a biological justification of this formulation, see Scudo (1964).

The first model treated here (due to FINNEY) is extremely simple. It is included for purposes of comparison with the later models. We allow three genotypes AA, $\mathrm{AB}$ and $\mathrm{BB}$, but the only matings producing viable offspring are those between a homozygote and a heterozygote. Alternatively, the genotypes can be partitioned as in Table 1, with matings possible only between members of different sets. If the frequencies of the $\mathrm{AA}, \mathrm{AB}$ and $\mathrm{BB}$ in the $n$th generation are respectively $u_{n}$, $v_{n}, w_{n}$ with $u_{0}, v_{0}, w_{0}$ being those in the initial population, we obtain the following recursion relations

$$
\begin{aligned}
\text { (i) } & T_{n-1} u_{n}=u_{n-1} v_{n-1} \\
\text { (ii) } & T_{n-1} v_{n}=u_{n-1} v_{n-1}+w_{n-1} v_{n-1} \\
\text { (iii) } & T_{n-1} w_{n}=w_{n-1} v_{n-1}
\end{aligned}
$$

where $T_{n-1}$ is a normalizing constant inserted to keep everything in terms of frequencies.

Obviously

$$
\frac{u_{n}}{w_{n}}=\frac{u_{n-1}}{w_{n-1}}=\cdots=\frac{u_{0}}{w_{0}}=p, \text { say, }
$$

and $v_{n}=1 / 2$ for $n \geq 1$. It follows that

$$
u_{n}=\frac{p}{2(1+p)}, \quad w_{n}=\frac{1}{2(1+p)} \quad \text { for } n \geq 1 .
$$

Thus, after one generation, the population has reached one of a possible continuum of equilibrium points, the exact point depending on the initial constitution of the population. (It is interesting to compare this result with the HARDY-WEINBERG law.)

Significant changes occur when a third allele is incorporated into the above model. We consider two cases according to whether the third allele $\mathrm{C}$ is introduced

TABLE 1

Model I

$\mathrm{AA}^{\text {Set } 1} \mathrm{BB} \quad \begin{gathered}\text { Set } 2 \\ \mathrm{AB}\end{gathered}$


into set 1 (model II) or set 2 (model III). In model II the incompatibility is specified by the following table:

TABLE 2

Model II

\begin{tabular}{|c|c|c|c|c|c|c|}
\hline & \multicolumn{5}{|c|}{ Set 1} & \multirow{2}{*}{$\frac{\text { Set } 2}{\mathrm{AB}}$} \\
\hline genotype & $\mathrm{AA}$ & $\mathrm{BB}$ & $\mathrm{BC}$ & $\mathrm{AC}$ & CC & \\
\hline nth generation frequency & $u_{n}$ & $w_{n}$ & $x_{n}$ & $y_{n}$ & $z_{n}$ & $v_{n}$ \\
\hline initial frequency & $u_{0}$ & $w_{0}$ & $x_{0}$ & $y_{0}$ & $z_{0}$ & $v_{0}$ \\
\hline
\end{tabular}

Once again matings are considered to take place only between individuals in different sets. The relations connecting genotype frequencies over successive generations are

$$
\begin{aligned}
& \text { (i) } T_{n-1} v_{n}=v_{n-1} u_{n-1}+v_{n-1} w_{n-1}+\frac{v_{n-1} x_{n-1}}{2}+\frac{v_{n-1} y_{n-1}}{2} \\
& \text { (ii) } T_{n-1} u_{n}=v_{n-1} u_{n-1}+\frac{v_{n-1} y_{n-1}}{2} \\
& \text { (iii) } T_{n-1} v_{n}=v_{n-1} w_{n-1}+\frac{v_{n-1}}{2} \frac{x_{n-1}}{2} \\
& \text { (iv) } T_{n-1} x_{n}=\frac{v_{n-1} x_{n-1}+v_{n-1} y_{n-1}}{2} \\
& \text { (v) } T_{n-1} y_{n}=\frac{v_{n-1} x_{n-1}+v_{n-1} y_{n-1}}{2} \\
& \text { (vi) } \quad z_{n}=0 \quad \text { for } n>1,
\end{aligned}
$$

where $T_{n-1}=2 v_{n-1}\left(1-v_{n-1}\right)$ is a normalizing factor. Notice that $u_{n}+w_{n}=v_{n}$ and $x_{n}=y_{n}$ for $n \geq 1$. Hence

and

$$
\frac{u_{n}}{x_{n}}=\frac{u_{n-1}}{x_{n-1}}+\frac{1}{2}=\frac{u_{n-2}}{x_{n-2}}+1=\cdots=\frac{n}{2}+\frac{u_{0}}{x_{0}}
$$

$$
\frac{w_{n}}{x_{n}}=\frac{n}{2}+\frac{w_{0}}{x_{0}} .
$$

Therefore $x_{n} \rightarrow 0$ and then $y_{n} \rightarrow 0$, so that $u_{n}+v_{n}+w_{n}=2 v_{n} \rightarrow 1$ or $v_{n} \rightarrow 1 / 2$. Since

$$
\frac{u_{n}}{w_{n}}=\frac{\frac{u_{0}}{x_{0}}+\frac{n}{2}}{\frac{w_{0}}{x_{0}}+\frac{n}{2}} \longrightarrow 1 \quad \text { as } n \rightarrow \infty
$$

we have $u_{n} \rightarrow 1 / 4, w_{n} \rightarrow 1 / 4$. The ultimate structure of the population is therefore $u_{e}=w_{e}=1 / 4, v_{e}=1 / 2$ and is independent of the initial makeup of the population.

It is intuitively obvious that the allele $C$ will eventually disappear. It is not evident that the addition of the third allele fundamentally alters the potential equilibrium configuration of the population. In fact, the previous continuum of fixed points is reduced to the single point $u_{e}=w_{e}=1 / 4, v_{e}=1 / 2$. What is perhaps 
more important is that the rate at which the equilibrium is approached is drastically altered. In model I the equilibrium point (which depends on the initial conditions) is achieved in one generation. In model II the third allele disappears at an algebraic rate (i.e., the frequencies of the genotypes $\mathrm{BC}$ and $\mathrm{AC}$, $x_{n}$ and $y_{n}$ in the $n$th generation, diminish like $1 / n$ as $n$ increases) which means that the equilibrium frequencies are attained very slowly; in terms of the allele $\mathrm{C}$, its frequency takes approximately 100 generations to decrease to $1 \%$ and 1000 generations to decrease to $0.1 \%$.

This reduction in the rate of approach to the equilibrium point seems to us to have some biological significance. Firstly, in a multiallelic system obeying similar mating laws to those postulated here, it can be inferred that although an allele (like C) may eventually disappear it will exist in the population for long periods of time. Conceivably, over this long duration, environmental or other changes could occur which might then give the allele in question an advantage, permitting it to become established. Secondly, if one observes a multiallelic population such as that in the second model it is quite likely that the observation will be made while the population is in a state of transition to a configuration containing far fewer alleles than actually noticed.

The incorporation of the third allele into model I to form model II causes profound changes in the equilibrium behavior, as shown above. It is interesting and $a$ priori not obvious that these changes are influenced by the manner in which the third allele is introduced. The third model, specified by Table 3, illustrates this point. Again matings are permitted only between members of different sets. The only change from model II is the set to which $\mathrm{C}$ has been added. It is possible to maintain a status quo with, for example, only $A A$ in Set 1 and $A B$ and $A C$ in Set 2 . In fact there are three families of equilibrium points, and the initial conditions determine which is reached. The equilibrium behavior differs profoundly from that of the previous model. The following is a brief discussion of the mathematical results obtained. The mathematics is more complicated, but since it may be of use in the analysis of other genetic models, it has been included in the Section "Analysis of Model III."

From Table 3 the recursion relations connecting the genotype frequencies over successive generations are

$$
\begin{aligned}
\text { (i) } & T_{n-1} u_{n}=u_{n-1}\left(v_{n-1}+y_{n-1}\right) \\
\text { (ii) } & T_{n-1} w_{n}=w_{n-1}\left(v_{n-1}+x_{n-1}\right) \\
\text { (iii) } & T_{n-1} v_{n}=u_{n-1}\left(v_{n-1}+x_{n-1}\right)+w_{n-1}\left(v_{n-1}+y_{n-1}\right)
\end{aligned}
$$

\begin{tabular}{|c|c|c|c|c|c|c|}
\hline \multirow[b]{2}{*}{ genotype } & \multicolumn{2}{|c|}{ Set 1} & \multicolumn{3}{|c|}{ Set 2} & \multirow[b]{2}{*}{$\mathrm{CO}$} \\
\hline & $\mathrm{AA}$ & $\mathrm{BB}$ & $\mathrm{AB}$ & $\mathrm{AC}$ & $\mathrm{BC}$ & \\
\hline $\begin{array}{r}\text { nth generation frequencies } \\
\text { initial frequencies }\end{array}$ & $\begin{array}{l}u_{n} \\
u_{0}\end{array}$ & $\begin{array}{l}w_{n} \\
w_{0}\end{array}$ & $\begin{array}{l}v_{n} \\
v_{0}\end{array}$ & $\begin{array}{l}y_{n} \\
y_{0}\end{array}$ & $\begin{array}{l}x_{n} \\
x_{0}\end{array}$ & $\begin{array}{l}z_{n} \\
z_{0}\end{array}$ \\
\hline
\end{tabular}

TABLE 3

Model III 


$$
\begin{array}{rrrl}
\text { (iv) } & T_{n-1} y_{n}=u_{n-1}\left(y_{n-1}+x_{n-1}\right) \\
\text { (v) } & T_{n-1} x_{n}=w_{n-1}\left(y_{n-1}+x_{n-1}\right) \\
\text { (vi) } & z_{n}=0 \quad n \geq 1
\end{array}
$$

where $T_{n-1}=2\left(u_{n-1}+w_{n-1}\right)\left(v_{n-1}+x_{n-1}+y_{n-1}\right)$ is a normalizing factor.

From the relations (3) it is easy to establish three families of equilibrium points:

$$
\begin{array}{ll}
F_{1}: & w_{e}=1 / 2, v_{e}+x_{e}=1 / 2, \\
F_{2}: & u_{e}=1 / 2, v_{e}+y_{e}=1 / 2, \\
F_{3}: & u_{e}+w_{e}=1 / 2, v_{e}=1 / 2 .
\end{array}
$$

We prove that the vector $\left(u_{n}, w_{n}, v_{n}, x_{n}, y_{n}\right)$ always converges as $n \rightarrow \infty$, even though there exist three continua of equilibria. More importantly we have determined precise domains of attraction to the respective equilibria.

The task at hand is to prove convergence, and to ascertain which of these families of equilibria is reached. The result is sensitive to the nature of the initial frequencies. For example, if $u_{0} / w_{0} \leq 1, y_{0} / x_{0} \leq 1$ with $u_{0} y_{0} / w_{0} x_{0}<1$ then the population will ultimately reach a point belonging to the family $F_{1}$; the exact point depends on the precise initial conditions. The rate of approach, which is geometrically fast with ratio $2 v_{e}$, also depends functionally on the initial frequencies. ( $w_{n}$ converges to $1 / 2$ geometrically with rate $2 v_{e}$ means that for $n$ large enough $\left|w_{n}-1 / 2\right|$ decreases by a factor $2 v_{e}$ per generation. Generally geometric convergence is much faster than algebraic, especially for low frequencies. It is possible, however, if the rate is close to 1 , for geometric convergence to be quite slow. This is the case in Table 5. The limiting equilibrium always satisfies $v_{e}>0, x_{\mathrm{e}}>0$; which affirms specifically that all three alleles are always present. We establish in the following section, that whereas $v_{e}$ is bounded away from zero independently of the initial frequencies, $x_{e}$ can be arbitrarily small. This implies that the geometric rate of approach may involve a factor close to $1 \mathrm{in}$ which event the equilibrium is attained relatively slowly. Under the initial conditions $w_{0} / u_{0} \leq 1, x_{0} / y_{0} \leq 1$ and $w_{0} x_{0} / u_{0} y_{0}<1$ corresponding results obtain; convergence takes place to a point of $F_{2}$, with both the precise limiting equilibrium and the rate of approach depending on the initial conditions.

It is striking that a stable situation can exist in which one allele is present at an extremely small frequency. Table 5 describes a concrete example in which this case arises. The computation in Table 5 further demonstrates the care required in interpreting data. A cursory glance at the table might lead one to surmise that the genotype AC is disappearing when in fact it has become stabilized at a very low frequency. Of course the mathematical theory dictates that $x_{e}>0$ so that a correct interpretation of the table is unavoidable.

Now since $y_{n} / x_{n}=u_{n-1} / w_{n-1}$, the result described above also resolves the case where for some $n,\left(u_{n} / w_{n}-1\right)$ and $\left(u_{n+1} / w_{n+1}-1\right)$ are of the same sign (see Tables 4,5$)$. The remaining possibility is that $\left(u_{n} / w_{n}-1\right)$ is a sequence alternating in sign. In this case we establish (see "Analysis of Model III" below) convergence to the special equilibrium point $u_{e}=w_{e}=1 / 4, v_{e}=1 / 2$, a member of $F_{3}$, and convergence then occurs at an algebraic rate.

The genotype frequencies effectively entail 4 independent variables. Theoret- 
ical considerations indicate that the case of alternation mentioned previously is possible only if the initial vector of genotype frequencies belongs to a hypersurface of dimension less than 4 . The case $u_{0}=w_{0}, y_{0}=x_{0}$ (which implies $u_{\mathrm{n}}=w_{\mathrm{n}}$, $y_{n}=x_{n}$ for every $n$ ) can be regarded as a case of degenerate alternation.

In every case convergence takes place to one of the families $F_{1}, F_{2}, F_{3}$. From the previous paragraph, it is obvious that the initial conditions determine the precise equilibrium attained. This is in sharp contrast to model II where the single equilibrium $u_{e}=w_{e}=1 / 4, v_{e}=1 / 2$ is ultimately achieved albeit very slowly. On the other hand, by including the allele $C$ in the set with the heterozygote, the equilibrium $u_{e}=w_{e}=1 / 4, v_{e}=1 / 2$ seems to become almost irrelevant. It is surprising and somewhat puzzling that the change from model II to model III should alter the equilibrium behavior so profoundly.

Tables 4 and 5 illustrate concretely the dependence of the equilibrium behavior on the initial conditions.

Analysis of Model III. For a complete analysis of this third model we have to consider various types of initial conditions. Consider first the case

Then, from (3) (i) and (3) (ii),

$$
\frac{u_{0}}{w_{0}} \leq 1 \frac{\gamma_{0}}{x_{0}}<1 .
$$

$$
\frac{u_{n}}{w_{n}}<\frac{u_{n-1}}{w_{n-1}}
$$

so that $\left\{u_{n} / w_{n}\right\}$ is a bounded decreasing sequence of positive numbers and hence $u_{n} / w_{n}$ tends to a limit $\alpha \geq 0$. Since $y_{n} / x_{n}=u_{n-1} / w_{n-1}$ we have $y_{n} / x_{n} \downarrow \alpha$ also.

We now argue by contradiction; suppose $\alpha>0$, and consider

$$
\begin{aligned}
\frac{v_{n}}{x_{n}+y_{n}} & =\frac{v_{n-1}}{x_{n-1}+y_{n-1}}+\frac{u_{n-1} x_{n-1}+w_{n-1} y_{n-1}}{\left(u_{n-1}+w_{n-1}\right)\left(x_{n-1}+y_{n-1}\right)} \\
& =\frac{\frac{v_{n-1}}{x_{n-1}+y_{n-1}}+\frac{\frac{u_{n-1}}{w_{n-1}}+\frac{y_{n-1}}{x_{n-1}}}{\left(1+\frac{u_{n-1}}{w_{n-1}}\right)\left(1+\frac{y_{n-1}}{x_{n-1}}\right)} .}{} .
\end{aligned}
$$

But the last term on the right hand side is bounded away from zero, since $\alpha>0$. Hence there exists an integer $N$ and a positive constant $C$ such that for $n \geq N$, we have on iteration,

$$
\frac{x_{n}+\gamma_{n}}{v_{n}} \leq \frac{1}{n C+D(N)}
$$

where $D(N)$ is a constant depending only on $N$. This last term converges to zero as $n \rightarrow \infty$. Thus, $x_{n}+y_{n} \rightarrow 0$ so that $x_{n} \rightarrow 0, y_{n} \rightarrow 0$. Now

$$
\left|\frac{u_{n}+w_{n}-v_{n}}{y_{n}-x_{n}}\right|=\left|\frac{y_{n-1}-x_{n-1}}{y_{n-1}+x_{n-1}}\right| \leq 1 \text {, }
$$

and hence we may conclude that $u_{n}+w_{n}-v_{n} \rightarrow 0$. Moreover, since $u_{n}+w_{n}+$ $v_{n} \rightarrow 1$, we infer that $u_{n}+w_{n} \rightarrow 1 / 2, v_{n} \rightarrow 1 / 2$. Again, from (4) there exist positive constants $E$ and $F$ such that for $n$ large enough $\left(y_{n}+x_{n}\right) / v_{n} \geq(n E+F)^{-1}$. Since the harmonic series diverges and $v_{n} \rightarrow 1 / 2$, we have $\sum_{1}^{\infty}\left(y_{n}+x_{n}\right)=\infty$. Now 
TABLE $4^{*}$

An example of Model III with $\mathrm{u}_{1} / \mathrm{w}_{1}>1, \mathrm{u}_{2} / \mathrm{w}_{2}>1$ and fast convergence

\begin{tabular}{cccccc}
\hline Generation & $\begin{array}{c}\mathrm{AA} \\
u_{n}\end{array}$ & $\begin{array}{c}\mathrm{BB} \\
w_{n}\end{array}$ & $\begin{array}{c}\mathrm{AB} \\
v_{n}\end{array}$ & $\begin{array}{c}\mathrm{BC} \\
x_{n}\end{array}$ & $\begin{array}{c}\mathrm{AC} \\
y_{n}\end{array}$ \\
\hline$n=0$ & .300000 & .100000 & .200000 & .205000 & .195000 \\
1 & .246874 & .083437 & .335416 & .083333 & .249999 \\
2 & .326206 & .079747 & .344824 & .063480 & .185740 \\
3 & .358842 & .067511 & .363879 & .041207 & .168558 \\
4 & .390597 & .055908 & .370658 & .028951 & .153884 \\
5 & .414514 & .045200 & .375119 & .020681 & .144484 \\
6 & .433580 & .036014 & .377554 & .015028 & .137821 \\
7 & .448572 & .028382 & .378956 & .011050 & .133037 \\
8 & .460310 & .022185 & .379763 & .008196 & .129543 \\
$\vdots$ & & & & & \\
43 & .499996 & .000001 & .380870 & .000000 & .119130 \\
\hline
\end{tabular}

In this case convergence is at a geometric rate and the equilibrium lies in $\mathrm{F}_{2}$. Differences $<10^{-6}$ are neglected.

* We are indebted to Mr. Markus Nabholz for assistance with the computations of Tables 4 and 5.

TABLE 5

An example of Model III with $\mathrm{u}_{1} / \mathrm{w}_{1}<1, \mathrm{u}_{2} / \mathrm{w}_{2}<1$ and slow convergence

\begin{tabular}{rrrrrr}
\hline Generation & $\begin{array}{c}\mathrm{AA} \\
u_{n}\end{array}$ & $\begin{array}{c}\mathrm{BB} \\
w_{n}\end{array}$ & $\begin{array}{c}\mathrm{AB} \\
v_{n}\end{array}$ & $\begin{array}{c}\mathrm{BC} \\
x_{n}\end{array}$ & $\begin{array}{c}\mathrm{AC} \\
\gamma_{n}\end{array}$ \\
\hline$n=0$ & .20100000 & .19900000 & .20000000 & .21000000 & .19000000 \\
1 & .16331249 & .16997916 & .33337499 & .16583333 & .16749999 \\
2 & .18405958 & .19093572 & .37502031 & .12749234 & .12249203 \\
3 & .19535440 & .20468927 & .39997032 & .10182653 & .09815947 \\
4 & .20272563 & .21397645 & .41663077 & .08527812 & .08138900 \\
5 & .20768693 & .22092495 & .42852188 & .07336177 & .06950443 \\
6 & .21117187 & .22637185 & .43743947 & .064443901 & .06057777 \\
7 & .21366836 & .23082391 & .44437303 & .05749771 & .05363696 \\
8 & .21547356 & .23457858 & .44991802 & .05194527 & .04808454 \\
$\vdots$ & & & & & \\
50 & .19580405 & .29483422 & .48988814 & .01166596 & .00780761 \\
$\vdots$ & & & & & \\
500 & .01006937 & .48977055 & .49615028 & .00392839 & .00008139 \\
$\vdots$ & & & & & \\
1000 & .00021620 & .49978042 & .49615360 & .00384808 & .00000167 \\
$\vdots$ & & & & & \\
1250 & .00003137 & .49996813 & .49615360 & .00384664 & .00000024 \\
$\vdots$ & & & & & \\
1756 & .00000063 & .49999935 & .49615360 & .00384639 & .00000000 \\
\hline
\end{tabular}

In this case the equilibrium is in $F_{1}$. Note that $x_{\rho}$ is extremely small and that the geometric rate of convergence $\left(2 v_{\rho}\right)$ is very close to 1 . This accounts for the slow rate. Differences less than 10-8 are neglected. 
$\left[\left(x_{n}-y_{n}\right) /\left(\mathrm{x}_{n}+y_{n}\right)\right] \rightarrow(1-\alpha) /(1+\alpha)$ so that $\sum_{1}^{\infty}\left(x_{n}-y_{n}\right)=\infty$, also. An elementary calculation, using $(5)$, reveals that

$$
\frac{u_{n}}{w_{n}}=\frac{u_{n-1}}{w_{n-1}} \frac{\left(1+\frac{y_{n-1}}{v_{n-1}}\right)}{\left(1+\frac{x_{n-1}}{v_{n-1}}\right)}=\frac{u_{0}}{w_{0}} \prod_{k=1}^{n}\left(1-\frac{x_{k}-\gamma_{k}}{v_{k}}+0\left(\frac{1}{k^{2}}\right)\right)
$$

But since $v_{k}$ is bounded away from zero and $\Sigma\left(x_{k}-y_{k}\right)$ diverges, we invoke a well known theorem on infinite products to conclude that $u_{n} / w_{n} \rightarrow 0$ contradicting the original assumption. Therefore $u_{n} / w_{n} \rightarrow 0, y_{n} / x_{n} \rightarrow 0$ so that $u_{n} \rightarrow 0$, $y_{n} \rightarrow 0$. From equations (3), $u_{n}+w_{n} \leq 1 / 2$, and since $y_{n} \rightarrow 0$ we have $v_{n}+x_{n}$ $\geq 1 / 2$ for $n$ large enough. But

$$
\frac{x_{n}+v_{n}}{w_{n}}=\frac{u_{n-1}}{w_{n-1}}+1+\frac{2 y_{n-1}}{v_{n-1}+x_{n-1}} \rightarrow 1
$$

so that $w_{n} \rightarrow 1 / 2, v_{n}+x_{n} \rightarrow 1 / 2$. From (4) $\frac{y_{n-1}}{x_{n-1}}=\frac{u_{n-2}}{w_{n-2}}$ and the fact that $\left\{u_{n} / w_{n}\right\}$ is a decreasing sequence, we have, for every $k$,

$$
C\left(\frac{u_{k-1}}{w_{k-1}}+\frac{u_{k-2}}{w_{k-2}}\right) \leq \frac{v_{k}}{x_{k}+y_{k}}-\frac{v_{k-1}}{x_{k-1}+y_{k-1}} \leq \frac{u_{k-1}}{w_{k-1}}+\frac{u_{k-2}}{w_{k-2}}
$$

where $C=\left(1+\left(u_{0} / w_{0}\right)\right)^{-2}$. Summing over $k$, from 1 to $n$, we have

$$
C\left[2 \sum_{k=1}^{n-1} \frac{u_{k-1}}{w_{k-1}}+\frac{y_{0}}{x_{0}}\right]+\frac{v_{0}}{x_{0}+y_{0}} \leq \frac{v_{n}}{x_{n}+y_{n}} \leq \frac{v_{0}}{x_{0}+y_{0}}+2 \sum_{k=1}^{n} \frac{u_{k-1}}{w_{k-1}}+\frac{y_{0}}{x_{0}}
$$

From (8) we see that $v_{n}$ is bounded away from zero. If we can show that $\sum_{k}\left(u_{k} / w_{k}\right)<\infty$ then (8) would imply that $\lim _{n} v_{n} / x_{n}$ exists, and since $v_{n}+x_{n} \rightarrow 1 / 2$ we would have $\lim _{n} x_{n}>0$. We now prove that $\sum_{k} u_{k} / w_{k}<\infty$. Assume, to the contrary, that $\Sigma u_{k} / w_{k}=\infty$. From (3) (i) and (3) (ii) we have, with some rearrangement,

$$
\frac{u_{n}}{w_{n}}-\frac{u_{n-1}}{w_{n-1}}=\frac{\left(u_{n-1} / w_{n-1}\right)\left(u_{n-2} / w_{n-2}-1\right)}{\left(v_{n-1} / x_{n-1}\right)+1}
$$

Now $u_{n} / w_{n} \downarrow 0$. Hence for any $\varepsilon>0$ there exists $N$ such that for $n \geq N$

$$
\frac{u_{n}}{w_{n}}-\frac{u_{n-1}}{w_{n-1}} \leq(\varepsilon-1) \frac{\left(u_{n-1} / w_{n-1}\right)}{\left(v_{n-1} / x_{n-1}\right)+1} \text {. }
$$

Returning to (8) we see, since $\Sigma u_{k} / w_{k}$ diverges, that

$$
\frac{v_{n}}{x_{n}} \text { is of the order } \sum_{k=1}^{n} \frac{u_{k}}{w_{k}}
$$

Summing both sides of (9) over $n$, the sum of the left hand side is a telescoping series, and remains bounded, while the right hand side, by virtue of (10) and an elementary theorem on divergent series (i.e., $\Sigma a_{n}=\infty$ and $A_{n}=\sum_{k=0}^{n} a_{k}$ implies $\left.\Sigma\left(a_{n} / A_{n}\right)=\infty\right)$, diverges to $-\infty$. This produces the required contradiction so that $\Sigma u_{k} / w_{k}<\infty$ must prevail and consequently $x_{e}>0$.

We have therefore established that if $u_{0} / w_{0} \leq 1, y_{0} / x_{0} \leq 1, u_{0} y_{0} / w_{0} x_{0}<1$ then there is convergence to a member of the family $w_{e}=1 / 2 v_{e}+x_{e}=1 / 2$; $v_{e}>0, x_{e}>0$. By completely symmetrical considerations when $w_{0} / u_{0} \leq 1$, 
$x_{0} / y_{0} \leq 1, w_{0} x_{0} / u_{0} y_{0}<1$ the limit equilibrium has the form $u_{e}=1 / 2, v_{e}+y_{e}=$ $1 / 2 ; v_{e}>0, y_{e}>0$.

To obtain the rate of convergence to a fixed point of the form $w_{e}=1 / 2, v_{e}+x_{e}$ $=1 / 2$ we examine

$$
u_{n}=\frac{u_{n-1}\left(v_{n-1}+y_{n-1}\right)}{2\left(u_{n-1}+w_{n-1}\right)\left(v_{n-1}+x_{n-1}+y_{n-1}\right)} .
$$

But $y_{n} \rightarrow 0$ and $2\left(u_{n}+w_{n}\right)\left(v_{n}+x_{n}+y_{n}\right) \rightarrow 1 / 2$ so that

$$
\lim _{n \rightarrow \infty}\left(\frac{u_{n}}{u_{n-1}}\right)=2 v_{e}<1 \text {. }
$$

Hence the rate of convergence of $u_{n}$ is geometric with ratio of decrease approximately $2 v_{e}$ per generation. It is then not difficult to show that $w_{n} \rightarrow 1 / 2$ and $v_{n}+x_{n} \rightarrow 1 / 2$ with convergence occurring at a geometric rate.

The above analysis resolves the case where for some $n,\left(\left(u_{n-1} / w_{n-1}\right)-1\right)$ and $\left(\left(u_{n} / w_{n}\right)-1\right)$ are both nonzero and of the same sign. Consider now the circumstance that the signs of these two quantities alternate continually as $n \rightarrow \infty$. Suppose $u_{0} / w_{0}<1, y_{0} / x_{0}>1$. This of course means $y_{1} / x_{1}<1$ and $u_{1} / w_{1}>1$. Then $u_{1} / w_{1}=\left(y_{1} / x_{1}\right)\left(v_{0}+y_{0}\right) /\left(v_{0}+x_{0}\right)>1$. Obviously $\left(v_{0}+y_{0}\right) /\left(v_{0}+x_{0}\right)<y_{0} / x_{0}$ (since $\left.x_{0}<y_{0}\right)$ and therefore $\left(y_{1} / x_{1}\right)\left(y_{0} / x_{0}\right)>1$. Again

$$
\left.1>\frac{u_{2}}{w_{2}}=\frac{y_{2}}{x_{2}} \frac{\left(v_{1}+y_{1}\right.}{v_{1}+x_{1}}\right)>\frac{y_{2} y_{1}}{x_{2} x_{1}}
$$

Hence

$$
0>\frac{y_{2} y_{1}}{x_{2} x_{1}}-\frac{y_{0} y_{1}}{x_{0} x_{1}}=\frac{y_{1}}{x_{1}}\left(\frac{y_{2}}{x_{2}}-1-\frac{y_{0}}{x_{0}}+1\right),
$$

so that $y_{2} / x_{2}-1<y_{0} / x_{0}-1$. By induction $\left(y_{2 n} / x_{2 n}-1\right)$ is a decreasing sequence. Similarly $\left(1-y_{2 n+1} / x_{2 n+1}\right)$ is a decreasing sequence. Suppose that $\lim _{n \rightarrow \infty}\left(y_{2 n} / x_{2 n}\right)=1+\alpha$ and $\lim _{n \rightarrow \infty}\left(y_{2 n+1} / x_{2 n+1}\right)=1-\beta$ with $\alpha \geq 0, \beta \geq 0$. Then we can use equation (4) to assert that for $n$ large enough,

$$
\frac{v_{n}}{x_{n}+y_{n}} \geq \frac{v_{n-1}}{x_{n-1}+y_{n-1}}+\gamma
$$

where $\gamma$ is a positive constant. It follows that $v_{n} /\left(x_{n}+y_{n}\right) \geq n_{\gamma}$ and therefore $x_{n}+y_{n} \rightarrow 0$ as $n \rightarrow \infty$. From (6) we conclude that $v_{n} \rightarrow 1 / 2$.

Consider again

$$
\frac{u_{n}}{w_{n}}=\frac{u_{n-1}}{w_{n-1}} \quad\left(\frac{v_{n-1}+y_{n-1}}{v_{n-1}+x_{n-1}}\right)
$$

The second factor on the right tends to 1 and $y_{n} / x_{n}=u_{n-1} / w_{n-1}$. Passing to the limit in (12) and applying the findings of the previous paragraph, we obtain $1+\alpha=1-\beta$ and hence $\alpha=\beta=0$. This ensures that $\left|1-y_{n} / x_{n}\right| \rightarrow 0$. From (3) (i) and (3) (ii) we infer that $u_{n}-w_{n} \rightarrow 0$. Hence since $u_{n} / w_{n} \rightarrow 1$ the fixed point is $u_{e}=w_{e}=1 / 4, v_{e}=1 / 2$. Since the domains of attraction to stable sets must be open sets, general topological considerations dictate that the point $(1 / 4$, $1 / 2,1 / 4)$ is unstable in model III.

The very special case where $u_{0}=w_{0}, y_{0}=x_{0}$ is one in which the third allele disappears. In this case $u_{n}=w_{n}$ and $y_{n}=x_{n}$ so that

$$
\frac{v_{n}}{y_{n}}=n+\frac{v_{0}}{y_{0}} \text {. }
$$


Hence $y_{n} / v_{n} \rightarrow 0$ algebraically so that $y_{n} \rightarrow 0, x_{n} \rightarrow 0$. Also $\left(u_{n}+w_{n}\right) / v_{n}=1$ since $x_{n-1}=y_{n-1}$. Therefore $u_{n}+w_{n}+v_{n} \rightarrow 1$ with $u_{n}+w_{n} \rightarrow 1 / 2, v_{n} \rightarrow 1 / 2$ and since $u_{n}=w_{n}$ we must have $u_{e}=1 / 4, w_{e}=1 / 4, v_{e}=1 / 2$. Note that the rate of approach here is algebraic (see equation (13)). We have shown, therefore, that in every case convergence to an equilibrium point takes place.

\section{DISCUSSION AND SUMMARY}

The analysis itself has led to a number of conclusions which could not have been discerned solely by numerical computation.

(i) The addition of the third allele in models II and III could be expected to alter the equilibrium behavior from that in model I. However, the manner in which the allele is introduced influences this behavior in an unexpected way. In model II where the allele $\mathrm{C}$ is in the set with the two homozygotes $\mathrm{AA}$ and $\mathrm{BB}$ there is a single equilibrium, $1 / 4 \mathrm{AA}+1 / 2 \mathrm{AB}+1 / 4 \mathrm{BB}$, which is stable and is reached very slowly. When the allele $C$ is in the set with the heterozygote $A B$, three continuous families of equilibria result; $u_{e}=1 / 2, v_{e}+y_{e}=1 / 2 ; w_{e}=1 / 2$, $v_{e}+x_{e}=1 / 2 ; u_{e}+w_{e}=1 / 2, v_{e}=1 / 2$. The third continuum of equilibria effectively reduces to the single point $u_{e}=w_{e}=1 / 4, v_{e}=1 / 2$ in the sense that this is the only equilibrium of the family which can be attained. At points in the first two families, all three alleles are represented. The rate of approach to these equilibria is geometric with rate approximately $2 v_{e}$. The equilibrium $u_{e}=w_{e}=$ $1 / 4, v_{e}=1 / 2$ can be attained only if the initial frequency vector belongs to a lower dimensional hypersurface. It is therefore an unstable equilibrium in the classical sense. It is quite surprising that the seemingly small alteration of the basic model from II to III should be responsible for the marked differences in equilibrium behavior between the two models.

(ii) In model II (and in the degenerate case $u_{0}=w_{0}, y_{0}=x_{0}$ of model III) the allele $C$ disappears slowly. In most models where the mating system entails a form of selection, the rate of loss of an allele is very slow, usually no faster than algebraic. In the present context, this has two qualitative implications. First, the third allele will exist in the population for long periods and therefore be subject to environmental or other influences which could conceivably give it an advantage at a later stage such that this allele might then become established. The second implication is a more practical one. In the field several alleles may be observed at low and apparently constant frequencies. If the mating scheme of the population approximates that in the models here, it may be that the population is not at "polymorphic equilibrium" but in a state of slow transition to a configuration containing fewer alleles. This could explain the existence of a large number of alleles in a population. The analysis was carried out for the case of 3 alleles. The presence of a larger number of alleles in a population with this mating behavior would slow the approach to an equilibrium state even more.

(iii) In model III we have shown that it is possible to maintain a polymorphic equilibrium with the third allele, C, at an extremely small frequency. Such 
equilibria are usually attributed to a balance between selection and other pressures such as mutation.

(iv) It is evident from model III that in discussing equilibrium properties, numerical computations have to be used cautiously. In model III, for example, the precise fixed point depends on the initial conditions. We have given an example (Table 5) in which, at equilibrium, one genotype frequency is so small that at first glance it might be assumed to be effectively zero. However, the mathematical analysis establishes that this genotype frequency is positive at equilibrium.

In the paper, "Further Analysis of Negative Assortative Mating," another class of mating systems is examined. While we are most particularly interested in the qualitative findings outlined above, we believe that the mathematical approach used here may be applied profitably to other investigations of genetic models. There are, of course, many models which do not succumb to an analysis as complete as that available for the models considered here. For these computations can be of some help in indicating the transient behavior of the population. On the other hand wherever a mathematical analysis is possible, as in the models above, much more can be learned.

\section{SUMMARY}

In the usual two allele model with matings permissible only between homozygotes and heterozygotes it is well known that a continuum of equilibria exists. In this paper it is shown that the introduction of a third allele as originally proposed by Scudo produces either a single equilibrium or 3 families of equilibria depending on whether the genotypes containing the third allele are taken with the original homozygotes or heterozygote. Complete analyses of the models are given including the domains of attraction and rates of approach to the various equilibria. The type of mathematical analysis presented may prove useful in the treatment of other genetic models.

\section{LITERATURE GITED}

Bonmer, W. F., 1960 The genetics of homostyly in populations of Primula vulgaris. Phil. Trans. Roy. Soc. B 242 : $517-549$.

East, E. M., and A. J. Marjgelsdonf, 1925 A new interpretation of the hereditary behavior of self-sterile plants. Proc. Natl. Acad. Sci. 11: 166-171.

Finney, D. J., 1952 The equilibrium of a self-incompatible polymorphic species, Genetica 26: $33-64$.

KarLin, S., and M. W. Feldman, 1968 Further analysis of negative assortative mating. Genetics 59: 117-136.

NAYLOR, A. F., 1962 Mating systems which could increase heterozygosity for a pair of alleles, Am. Naturalist 96: 51-60.

Monan, P. A. P., 1962 The Statistical Processes of Evolutionary Theory. Clarendon Press, Oxford. 
Owen, A. R. G., 1953 A genetical system admitting of two distinct stable equilibria under natural selection. Heredity $7: 97-102$.

Scudo, F. M., 1964 Sex population genetics. Ric. Sci. (II-B) 34: 93-146.

Watterson, G. A., 1959 Non-random mating, and its effect on the rate of approach to homozygosity. Ann. Human Genet. 23 : 204-220.

Wonkman, P. L., 1964 The maintenance of heterozygosity by partial negative assortative mating. Genetics 50: 1369-1382. 